\title{
Correlation between Colloidal Stability and Surfactant Adsorption/Association Phenomena Studied by Light Scattering
}

\author{
Alessio Zaccone, Hua Wu, Marco Lattuada, and Massimo Morbidelli* \\ Institute for Chemical and Bioengineering, Department of Chemistry and Applied Biosciences, \\ ETH Zurich, 8093 Zurich, Switzerland
}

Received: September 21, 2007; In Final Form: November 4, 2007

\begin{abstract}
The stability of a colloidal system composed of styrene-acrylate copolymer particles and potassium stearate (KS) anionic surfactant molecules has been determined in terms of the Fuchs stability ratio, $W$, as a function of the surfactant concentration, by measuring the initial aggregation kinetics using the small-angle light scattering (SALS) technique. The structure of the particle surface is peculiar, being irregularly patterned, and thus represents a model system to investigate colloidal stability of nonsmooth colloidal particles. From the SALS kinetic experiments, it is found that the stability increases dramatically with KS concentration until the saturation of the available surface occurs. At concentrations higher than the saturation concentration, the $W$ value decreases markedly with $\mathrm{KS}$, as a consequence of attractive depletion forces induced by formation of micelles in the water phase. The adsorption isotherm, determined through the surface tension technique, agrees with the $W$ vs KS behavior, with respect to the onset of saturation and the surface-per-molecule value, and it can be described by the two-step Langmuir isotherm. Static light scattering spectra of the particles at different adsorbed amounts of KS have been fitted by means of the Lorenz-Mie theory and accounting for the experimentally determined particle size distribution. The increase in the particle diameter imputable to KS adsorption is sizable. Stability data measured under high fluid shear in a turbulent capillary (in the absence of any screening salt) fit well into this scenario. However, depletion forces are shown to be noncooperative with turbulent shear in the absence of screening electrolytes.
\end{abstract}

\section{Introduction}

Colloids and surfactants appear in a wide variety of natural phenomena and industrial applications. The latter category comprises, among others, the emulsion polymerization process, which is widely used to produce polymeric materials. In this process, surfactants are used to make possible the polymerization reaction. Several properties of the final product (i.e., the polymer colloidal particles) strongly depend on the surfactant type, its concentration, and its interactions with the monomer and with the polymer. ${ }^{1}$ Another example is given by protein-surfactant mixtures where the interaction of the protein with the surfactant can lead to protein unfolding, with substantial modification of the biological activity of the protein. ${ }^{2}$ Furthermore, surfactants are now being identified as a crucial agent for the synthesis and assembly of solution-grown semiconductor nanocrystals whose colloidal stability is controlled by the surfactant coating. ${ }^{3}$ Therefore, knowledge and understanding of phenomena involving surfactant in colloidal systems is crucial for the progress of many areas of application.

Mixtures of colloids and surfactants have been studied in the past with special attention either to their phase behavior or to the adsorption mechanisms and morphologies at the particles surface. ${ }^{4,5}$ Only very recently some experimental results have been published, which address the colloid stabilization/ destabilization effects induced by the surfactant in a more comprehensive way, i.e., including both adsorption (stabilizing processes) and depletion attractive forces induced by the surfactant micelles (destabilizing processes). ${ }^{6}$ However, although

* To whom correspondence should be addressed. E-mail: morbidelli@ chem.ethz.ch. Fax: 0041-44-6321082. this latter study gives compelling evidence of the phenomena involved, it is still far from providing a quantitative interpretation and understanding of such complex systems. In particular, the stability data as measured by the light-scattering technique should be regarded as being rather qualitative in that, in order to monitor the initial aggregation kinetics, it has been relied entirely on the detection of the linear-to-nonlinear transition of the intensity of light scattered at a single angle. Moreover, these latter authors studied a colloidal system of polystyrene particles polymerized in the absence of emulsifier, thus implying a high density of fixed-charges at the surface which dim the effect of surfactant adsorption on stability. Finally, most of the previously cited works deal with ideal, i.e., smooth and regular, colloid surfaces. This is however a recurrent case neither in nature nor in applications, where the surface of colloidal particles are invariably affected by heterogeneities of both a topological (roughness) and physicochemical (charge distribution) nature. ${ }^{7,8}$

Scattering techniques are widely used in order to investigate the surface properties of dispersed systems. ${ }^{9}$ However, despite the large amount of literature on this type of characterization using neutrons ${ }^{10}$ and $\mathrm{X}$-ray, the number of studies where visible light radiation has been used is more limited. Among those reporting on dynamic light scattering (DLS) characterization of adsorbate layers, we mention the work of Zhao and Brown, ${ }^{11}$ who used DLS to measure the growth in thickness of SDS adsorbate layers on polystyrene particles. They observed a dramatic increase in the hydrodynamic radius of the particles upon increase in the SDS concentration, up to a maximum increase of the radius of about $20 \mathrm{~nm}$ (relative to particles of about $200 \mathrm{~nm}$ in diameter). ${ }^{11}$ This result could only be explained 
by referring to multilayer micellization phenomena at the surface but yet it does not sound realistic that such a massive decoration of the particles could take place when considering the relatively small size of the SDS molecule. Moreover, such huge surface micellization phenomena are not reflected in the adsorption isotherm. In general, however, DLS measurements present the disadvantage of being highly dependent upon the position of the shear plane which is usually not known and which does not necessarily correspond to the interface between adsorbed layer and liquid phase. A combination of static light scattering (SLS) and DLS was used by $\mathrm{Wu}^{12}$ to clarify the structure of latex particles produced by microemulsion polymerization. This led to the formulation of the mostly accepted "core-shell" model for these latices, ${ }^{13}$ where half of the hydrophobic tail of the surfactant molecules is buried inside the polymer particle while the other half protrudes into the water phase together with the polar head.

The only study, to our knowledge, where SLS has been applied to the investigation of adsorbed layers is the one made by Yang et al. ${ }^{14}$ They employed a core-shell model to evaluate the growth of grafted layers during polymerization. However, since they used the Rayleigh theory to fit the experimental data, whereas the size of their particles was relatively large (and in some case even several times larger than the wavelength of the laser) and moreover without accounting for the polydispersity, the agreement between experiments and theory is only qualitative.

In the present work we report on an experimental study of the stability of a colloid-surfactant mixture spanning a broad range of surfactant concentrations. The purpose is to correlate the stability behavior with surface phenomena, mainly with the surfactant adsorption, in the range of concentrations up to the complete coverage of the surface (adsorption controlled regime). In order to better understand the transition from this latter regime to the depletion induced coagulation regime, it was necessary to characterize the adsorption mechanism quantitatively by means of the adsorption isotherm.

Furthermore, knowledge about the morphology of the adsorbed layer has been sought by means of SLS. The LorenzMie theory and the particle size distribution (PSD) measured from scanning electron microscopy (SEM) images have been combined to this purpose, attempting to determine the increase in particle diameter brought about by surfactant adsorption.

Finally, high-shear flow coagulation experiments in the absence of electrolyte have been carried out in a capillary. Colloid-surfactant mixtures at different surfactant concentration have been sheared and subsequently analyzed ex situ by means of the small-angle light scattering (SALS) technique in order to quantify the extent of aggregation phenomena induced by the high shear rate. Also in this case, a correlation between the behavior of the surfactant and the colloidal aggregation has been sought which consolidates the conclusions drawn by means of the other techniques employed and unfolds new issues related to stability of colloid-micelles mixtures under shear.

\section{Experiments and Methodology}

The Colloidal System and Sample Preparation. The colloidal dispersion is a styrene-acrylate copolymer latex produced by emulsion polymerization. The particles are spherical, as shown in Figure 1a, but they exhibit an irregularly grainypatterned surface with protruding grains separated by depressions. This is evident in Figure 1b.

The particles are polymerized in the presence of the same surfactant potassium stearate $\left(\mathrm{KS}, \mathrm{CH}_{3}\left(\mathrm{CH}_{2}\right)_{16} \mathrm{COOK}\right.$, Acros
Organics) used for our investigation. KS phase behavior is very rich, and it has been studied for a long time. Gregory and $\operatorname{Tartar}^{15}$ already reported about the practical impossibility of detecting the critical micelle concentration $(\mathrm{cmc})$ in the aqueous phase due to the high hydrophobic character of the molecule. Vincent and Skoulios ${ }^{16,17}$ analyzed the mesoscopic structures of aqueous gels formed by KS in solution by X-ray diffraction methods and measured the geometrical parameters of bilayers, determining the surface occupied by a molecule in close-packed structures to be of the order of $23 \AA^{2}$. More recently, Jenkins and Weiss ${ }^{18}$ got more insight into the phase behavior of KS by means of fluorescence spectroscopy and established that stearate anions associate into cylindrical micelles of undefined length. These micelles, with increasing concentration, then, form liquid crystalline phases prior to assembling into more ordered phases (arrays) and forming, finally, bilayer gels. They also give a measure of the length of a molecule in the stretched configuration of about $25.2 \AA(2.52 \mathrm{~nm})$. Given the scarce solubility of this surfactant in water at room temperature, for the adsorption experiments it was necessary to add it to the colloidal dispersion at $65{ }^{\circ} \mathrm{C}$ and keep the samples at the same temperature for several hours in order to ensure complete dissolution of the surfactant. Subsequently, the samples were allowed to equilibrate at $25{ }^{\circ} \mathrm{C}$ for at least $6 \mathrm{~h}$ prior to executing any kind of measurements. The low solubility of $\mathrm{KS}$ at room temperature offers the advantage of having practically no desorption of the surfactant upon dilution so that, after the adsorption equilibrium has been reached at room temperature, the surfactant molecules remain like "attached" to the particles surface. This has been verified by $\zeta$-potential measurements revealing that the $\zeta$-potential of the particles in the absence of salt remains unchanged upon diluting several times the original concentration even after 1 day.

The average diameter of the particles has been measured by image data analysis (IDA) from the SEM images and in water by SLS. In the first case, the size of about 200 particles has been measured. The experimental data have been best fitted by means of a log-normal pdf. The mean value of the log-normal pdf is: $\mu=\log R_{\mathrm{p}, \mathrm{m}}=6.3508 \pm 0.0058$, thus resulting in a mean diameter $\left(R_{\mathrm{p}}\right)$ equal to $573 \mathrm{~nm}$. The geometric mean standard deviation, $\sigma$, is 0.0376 . The experimental and the calculated distributions are shown in Figure 2. In the second case, a value of the diameter equal to about $578 \mathrm{~nm}$ has been found. This is quite in good agreement with the SEM analysis, and the small difference is imputable to the swelling effect of the water on the polymer chains at the interface (also notice that the acrylate groups are slightly polar), which is absent under vacuum conditions.

To investigate the system at different concentrations of $\mathrm{KS}$, it was necessary to remove from the latex the surfactant used for the polymerization process as well as the residual initiator. This has been done by mixing the latex with a mixture of cationic and anionic ion-exchange resins (Dowex MR-3, SigmaAldrich). The removal of ionic species was monitored by measuring the conductivity, which falls down by two orders of magnitudes as a consequence of the ions withdrawal, and it was assumed that the complete removal is achieved when the conductivity of the solution ceases to decrease. Since the resin used is made of polyelectrolyte chains adsorbed on solid porous beads, the possibility of leakage of polyelectrolyte species in the solution has been considered. To verify that no leakage occurred, DLS measurements on the water phase of the washed sample after centrifugation have been done in order to detect eventual presence of such macromolecules. The signal and the 


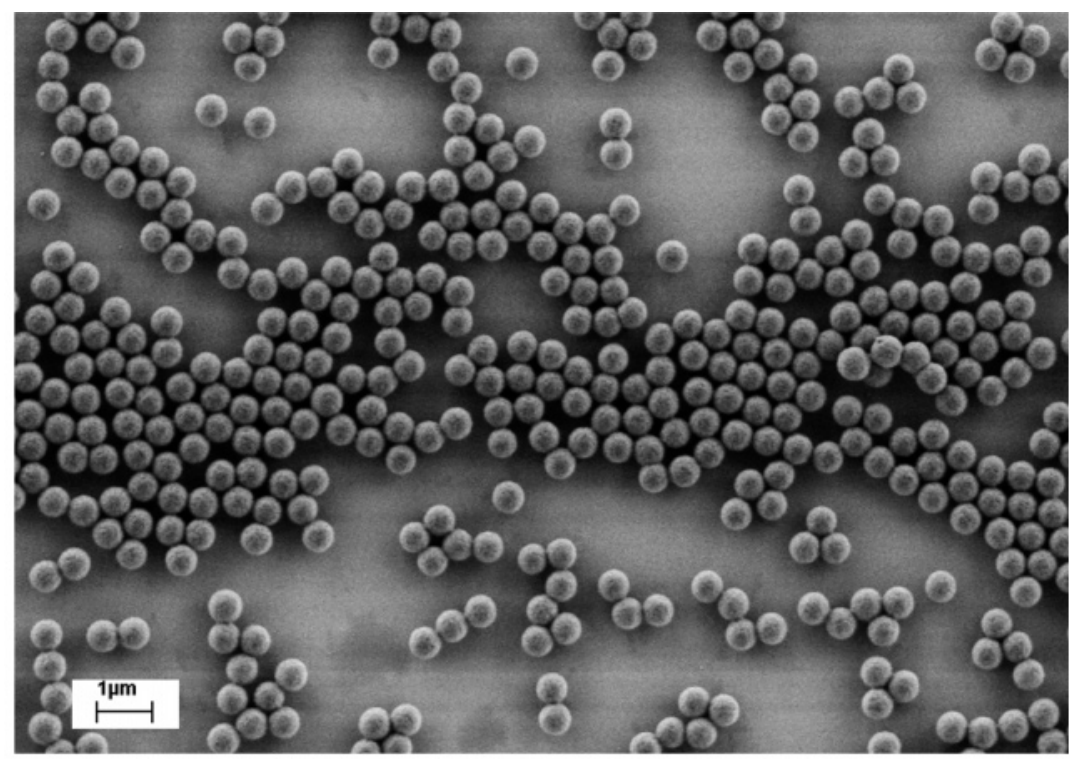

(a)

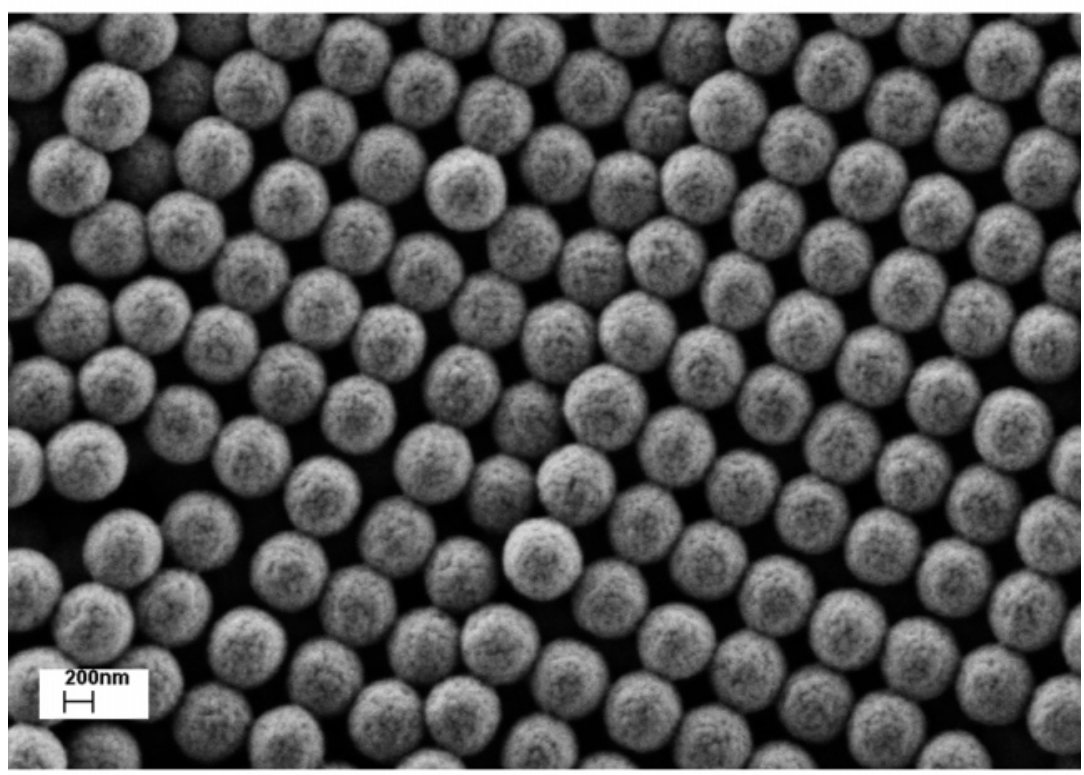

(b)

Figure 1. (a) SEM pictures of the colloidal particles. (b) the same particles seen with higher resolution SEM, showing the detail of the irregularly patterned surface.

intensity recorded are undistinguishable from those characteristic of demineralized water, thus excluding the occurrence of such kind of leakage.

Determination of Fuchs Stability Ratio by Measuring Doublet Formation Rate Using SALS. The primary particle aggregation or doublet formation rate is one of the fundamental features of the stability of a colloidal system. In particular, the doublet formation rate constant, $K_{1,1}$, is directly related to the so-called Fuchs stability ratio, $W$, through the simple expression: $K_{1,1}=K_{\mathrm{B}} / W$, where $K_{\mathrm{B}}(8 k T / 3 \eta$, where $k$ is Boltzmann's constant, $T$ is the absolute temperature, and $\eta$ is the viscosity of the liquid) is the Smoluchowski rate constant. ${ }^{19}$

The Fuchs stability ratio plays an important role also in the quantitative simulation of aggregation kinetics, which is usually

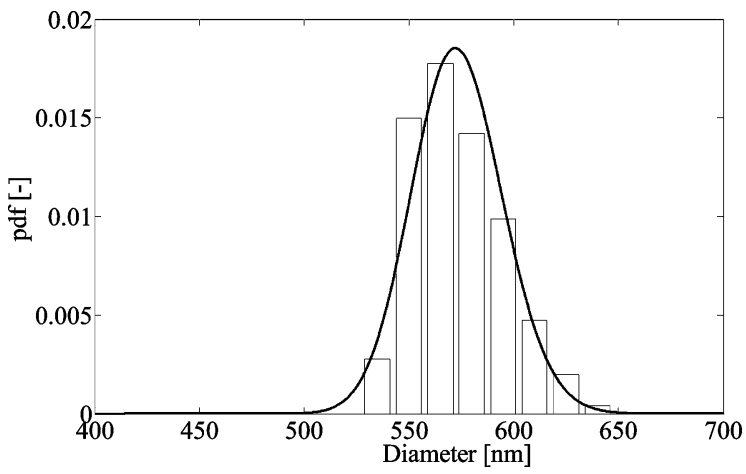

Figure 2. Experimental pdf (bars) and log-normal pdf fitted curve (continuous line). 
done using the Smoluchowski kinetic model based on the following population balance equation, written in discrete form ${ }^{21}$

$$
\frac{d N_{i}(t)}{d t}=-\sum_{j=1}^{\infty} K_{i, j} N_{i}(t) N_{j}(t)+\frac{1}{2} \sum_{j=1}^{i-1} K_{i-j, j} N_{i-j}(t) N_{j}(t)
$$

where $N_{i}(t)$ is the number concentration of clusters containing $i$ primary particles at time $t$ and $K_{i, j}$ is the aggregation rate constant (or kernel) between two clusters containing $i$ and $j$ primary particles, respectively, which is a complex function of various quantities including the Fuchs stability ratio $(W)$. In the initial stage of the aggregation, the conversion or fraction of primary particles forming doublets is defined as

$$
x=\frac{N_{1,0}-N_{1}}{N_{1,0}}
$$

where $N_{1,0}$ indicates the number of primary particles at time $t$ $=0$ and $N_{1}$ the number of particles at time $t$. Thus, in the initial stage of aggregation, the population balance equation, eq 1 , can be simplified to: $-d N_{1} / d t=K_{1,1} N_{1}{ }^{2}$. This, when integrated, yields: $1 / N_{1}-1 / N_{1,0}=K_{1,1} t$. Substituting eq 2 into the latter expression for $N_{1}$, we end up with

$$
\frac{x}{1-x}=N_{1,0} K_{1,1} t
$$

Hence, by plotting $x /(1-x)$ against $t$ in the aggregation stage where the conversion $x<20 \%$, one can obtain a straight line, from which the slope $\left(N_{1,0} K_{1,1}\right)$ gives the $K_{1,1}$ value. Deviation from the linearity indicates that the condition that the system is dominated by only primary particles and doublets is no longer satisfied. Now, the question is how to measure the conversion $x$ as a function of time. This is done in this work through the SALS technique.

The light-scattering technique measures the light intensity scattered by a system as a function of the scattering angle, $I(q)$, which, for a colloidal aggregating system under dilute conditions, is given by ${ }^{22}$

$$
I(q)=\frac{\sum_{i=1}^{\infty} N_{i} i^{2} S_{i}(q) P(q)}{\sum_{i=1}^{\infty} N_{i} i^{2}}
$$

where $q$ is the wave vector defined as: $q=4 \pi n / \lambda_{0} \sin (\theta / 2)$. $\lambda_{0}$ is the wavelength of the incident light, $n$ the refractive index of the surrounding medium, and $\theta$ the scattering angle. The other parameters and variables in eq 4 are the number $N_{i}$ and structure factor $S_{i}(q)$ of clusters with $i$ primary particles and the form factor of the primary particles, $P(q)$. Note that eq 4 has been written in a normalized form such that $I(q) \rightarrow 1$ as $q \rightarrow 0$. Moreover, when eq 4 is divided by the form factor, $P(q)$, an average structure factor, $\langle S(q)\rangle$, may be defined as follows

$$
\langle S(q)\rangle=\frac{I(q)}{P(q)}=\frac{\sum_{i=1}^{\infty} N_{i} i^{2} S_{i}(q)}{\sum_{i=1}^{\infty} N_{i} i^{2}}
$$

In the very initial stage of the aggregation the system can be regarded as consisting of only primary particles and doublets. In this case, we have that ${ }^{20}$

$$
\langle S(q)\rangle=1-\frac{x}{1+x}\left[1-\frac{\sin \left(2 q R_{\mathrm{p}}\right)}{2 q R_{\mathrm{p}}}\right]
$$

Therefore, when the measured $\langle S(q)\rangle$ is plotted against [1 $\left.\sin \left(2 q R_{p}\right) /\left(2 q R_{p}\right)\right]$, one obtains a straight line, whose slope gives an estimate of the conversion of primary particles to doublets, $x$. The so obtained $x$ values plotted as a function of time, as indicated by eq 3 , allow one to determine the rate constant for the doublet formation, $K_{1,1}$. The method is indeed very accurate and fully quantitative. ${ }^{18}$

A SALS instrument, Mastersizer 2000 (Malvern, U.K.), has been used to monitor the initial rate of aggregation. The scattering angle ranges from 0.02 to $40^{\circ}$, and the wavelength of the laser beam is $\lambda_{0}=633 \mathrm{~nm}$. A more detailed description of the instrument can be found in another contribution. ${ }^{23}$

The colloidal dispersion and the electrolyte solution were mixed, and samples were taken at different times by immediately diluting into $200 \mathrm{~mL}$ of water so as to quench the aggregation and to reach the volume fraction for the SALS measurement required to avoid multiple scattering effects. All experiments have been carried out at volume fraction $\phi=5 \times 10^{-3}$ and with $15 \mathrm{mM}$ of $\mathrm{Mg}_{2} \mathrm{SO}_{4}$ to induce aggregation in the presence of potential energy barrier (RLCA conditions). The total particle number concentration was then $N_{1,0}=5 \times 10^{16} \mathrm{~m}^{-3}$. The $\mathrm{pH}$ was measured prior to adding the electrolyte and was always found to be about 8, due to the weak basic character of KS. Only in the case of bare particles, due to the absence of KS, was the $\mathrm{pH}$ lower. In this case, an additional experiment was done by tuning the $\mathrm{pH}$ with a buffer $\left(\mathrm{NaHCO}_{3}, 3 \mathrm{mM}\right)$ in order to keep it equal to 8 . In this particular case the concentration of $\mathrm{MgSO}_{4}$ used was lower so as to keep the ionic strength constant and equal to that of all the other experiments.

Characterization of the Surface Surfactant Adsorption by Means of SLS. The general theory of light scattering by particles of any size and shape is referred to as the LorenzMie theory. On the basis of this theory, for a fixed wavelength of the incident radiation $\lambda_{0}$, the form factor of the primary particles is given by the scattered irradiance which, if the incident light is polarized parallel to the scattering plane (i.e., for vertically polarized incident light as in our case), reads ${ }^{24}$

$$
\begin{array}{r}
P\left(\theta, R_{\mathrm{p}}\right) \doteq I_{/ /}=\left(\frac{\lambda_{0}{ }^{2}}{4 \pi^{2} r^{2}}\right)\left\{\sum _ { n = 1 } ^ { \infty } \frac { 2 n + 1 } { n ( n + 1 ) } \left[a_{n} \pi_{n}(\cos \theta)+\right.\right. \\
\left.\left.\quad b_{n} t_{n}(\cos \theta)\right]\right\}^{2}
\end{array}
$$

The scattering coefficients $a_{n}$ and $b_{n}$, which are functions of the radius of the particles as well as of their refractive index, involve Riccati-Bessel functions and their derivatives, while $\pi_{n}$ and $\tau_{n}$, which are functions of the scattering angle, are expressed in terms of the Legendre polynomials and their derivatives. As we can see from eq 7 , for a fixed $\lambda_{0}$ and distance from the detector $r$, the form factor as predicted by the LorenzMie theory is a function of the following physical systemdependent parameters only: refractive index of the particles (implicit in the scattering coefficients $a_{n}$ and $b_{n}$ ) and radius of the particles. Clearly, if the refractive index of the particles is known, the radius of the particles can be used as the unique fitting parameter so as to find the best fit with the experimental 


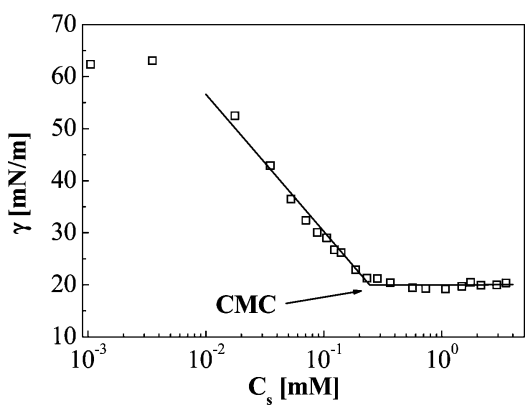

Figure 3. Surface tension $(\gamma)$ measured at $T=65{ }^{\circ} \mathrm{C}$ plotted as a function of surfactant concentration $\left(C_{s}\right)$. The knee indicated by the arrow is the cmc at $T=65^{\circ} \mathrm{C}$.

spectrum and thus determine the mean radius of the particles. This is a typical inverse scattering problem for determining the structure of dispersed particles. ${ }^{25}$

However, our system has a finite polydispersity, as will be shown below, that has to be accounted for appropriately. For this purpose, the experimental PSD obtained from image analysis of SEM pictures has been compounded into the inverse scattering problem so as to accurately determine the diameter of the particles in solution at various amounts of surfactant. As for many other systems, the two-parameter analytical pdf providing the best fit to the SEM data turned out to be the lognormal pdf

$$
p\left(R_{\mathrm{p}}\right)=\frac{1}{(2 \pi)^{1 / 2} \sigma R_{\mathrm{p}}} \exp \left[-\frac{\left(\log R_{\mathrm{p}}-\log R_{\mathrm{p}, \mathrm{m}}\right)^{2}}{2 \sigma^{2}}\right]
$$

where $\sigma$ is the geometric standard deviation and $\log R_{\mathrm{p}, \mathrm{m}}$ is the median value of the pdf. It follows that, rigorously, one has to compare the experimental spectrum with the following theoretical expression for the form factor of the particles

$$
\left\langle P\left(q, R_{\mathrm{p}}\right)\right\rangle=\int_{0}^{\infty} P\left(q, R_{\mathrm{p}}\right) p\left(R_{\mathrm{p}}\right) d R_{\mathrm{p}}
$$

where $P\left(q, R_{\mathrm{p}}\right)$ is the form factor as given by the Lorenz-Mie theory for a given particle radius $R_{\mathrm{p}}$ (eq 7) and $p\left(R_{\mathrm{p}}\right)$ is the analytical pdf expressing the PSD as given by the best fit of eq 8 to the measured SEM data.

The light-scattering instrument used for this characterization is the BI-200SM (Brookhaven), with a solid-state system laser (Ventus 532-500, Laser Quantum), operating at a wavelength of $\lambda_{0}=532 \mathrm{~nm}$, and a goniometer in the angle range of 15$150^{\circ}$. For each sample at least three repetitions of SLS measurements were carried out, and their averages are reported in the following. In order to have a more resolved contour at the angles corresponding to the intensity minima, the intensity of demineralized water was measured prior to measuring the sample and subsequently subtracted from the measured intensity of radiation scattered by the sample.

Adsorption Isotherm and $\zeta$-Potential Measurements. The adsorption isotherm of KS on the particles has been obtained by following a quite standard procedure, i.e., by means of surface tension measurements. The adsorption experiments were carried out as described above, and equilibrium was reached at $25^{\circ} \mathrm{C}$ for all samples. The particles were then centrifuged away and the surface tension of the supernatant analyzed by means of the Du-Nouy ring method (Kruss, K11 Tensiometer). The calibration curve relative to $\mathrm{KS}$, as obtained at $65^{\circ} \mathrm{C}$ to avoid precipitation at higher concentration, is shown in Figure 3. Note the quite sharp knee characteristic of transition to the micellar regime $(\mathrm{cmc})$, indicating good grade of the surfactant.
Aiming at a quantitative test of the surfactant-controlled stability scenario emerging from the SALS analysis, the experimental adsorption isotherm of the surfactant on the colloidal particles has been compared with theoretical predictions. For the latter ones, the so-called "L-S" or "two-step" adsorption isotherm proposed by $\mathrm{Gu}$ and $\mathrm{Zhu}$ has been used. ${ }^{26}$ The model was derived from equilibrium considerations, and the traditional approach of considering the reactions between free sites at the particle surface and the surfactant molecules was adopted. The first adsorption step, according to this theory, corresponds to the adsorption of single molecules that attach to the surface with the hydrophobic alkyl chains aligned parallel to the surface. At a certain critical concentration, the molecules start to interact with each other by means of lateral attractive forces, thus forming two-dimensional surface aggregates (hemimicelles). ${ }^{27}$ In particular, the previously singularly adsorbed molecules act as anchors to which $n-1$ molecules will attach to form an assembly of $n$ molecules. This second step corresponds to a sharp increase in the adsorption density. The mathematical form of the L-S adsorption isotherm is as follows ${ }^{26}$

$$
\Gamma=\frac{\Gamma_{\infty} k_{1} C_{S, e q}\left(\frac{1}{n}+k_{2} C_{S, e q}{ }^{n-1}\right)}{1+k_{1} C_{S, e q}\left(1+k_{2} C_{S, e q}^{n-1}\right)}
$$

where $C_{S, e q}$ is the bulk surfactant equilibrium concentration, $k_{1}$ and $k_{2}$ are the equilibrium constants for the two steps, while $\Gamma$ and $\Gamma_{\infty}$ are the equilibrium and the saturation adsorption densities at the solid/liquid interface, respectively. Physically relevant information can be inferred from the comparison with the theory, such as the mean aggregation number of the hemimicelles $(n)$, the effective surface-per-molecule at saturation (from $\Gamma_{\infty}$ ), and the onset of saturation (from the transition to the saturation plateau).

The $\zeta$-potential and the electrophoretic mobility of particles bearing different amounts of surfactant have been measured by means of a laser Doppler velocimetry device (ZetaSizer Nano, Malvern). To obtain a sufficiently clear signal and reproducible measurements, a volume fraction of $\phi=5 \times 10^{-4}$ has been employed, in the presence of the same concentration of $\mathrm{Mg}_{2}-$ $\mathrm{SO}_{4}(15 \mathrm{mM})$ used for the stability experiments.

Shear Flow Aggregation Experiments. Aiming at inducing colloidal aggregation in the absence of screening electrolytes with varying surfactant concentration, a capillary device has been constructed in order to generate a high-shear turbulent flow with steep turbulent velocity gradients. The capillary (length $L$ $=60 \mathrm{~mm}$, radius $R=0.25 \mathrm{~mm}$ ) has a curvilinear shape with a radius of curvature of $4.3 \mathrm{~mm}$. The curvature induces higher energy dissipation when compared to straight channels due to secondary flow taking place in the cross section of the capillary (the so-called Dean's flow). This secondary flow is also responsible for the increased residence time inside the shearing zone, which, otherwise, would be practically instantaneous. This is reflected in a characteristic curve for the capillary given by: $v=1.50(\Delta P / L)^{0.487}$, where $\Delta P$ is the pressure drop across the capillary and $v$ is the fluid velocity inside the capillary. The power-law exponent is lower than the value 0.534 given by the White empirical correlation for turbulent flow in straight tubes, thus indicating a significant extent of additional energy dissipation compared to the straight channel case. In order to generate turbulence inside the capillary, a piston pump driven by compressed air (M-110Y, Microfluidics) has been connected to the inlet of the capillary. The pressure at the inlet of the capillary has been measured by means of an electronic pressure 


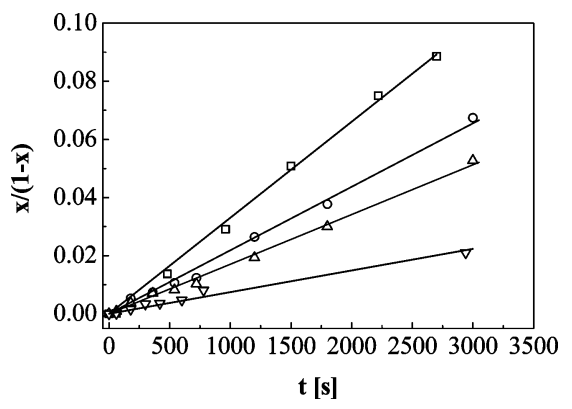

Figure 4. Conversion of the primary particles to doublets as a function of time at different total concentration of surfactant, $C_{s}$. ( $\left.\square\right) C_{s}=0.088$ $\mathrm{g} / \mathrm{dm}^{3} ;(\nabla) C_{s}=0.097 \mathrm{~g} / \mathrm{dm}^{3} ;(\Delta) C_{s}=0.1064 \mathrm{~g} / \mathrm{dm}^{3} ;(O) C_{s}=0.116$ $\mathrm{g} / \mathrm{dm}^{3}$. Straight lines are linear fit to the experimental data.

transducer. The flow rate was fixed to $Q=10 \mathrm{~mL} \mathrm{~s}^{-1}$ for all experiments, thus resulting in a shear rate at the wall of $\dot{\gamma}=$ $4 Q / \pi R^{3}=8.15 \times 10^{5} \mathrm{~s}^{-1}$. This value has to be taken as indicative, since turbulence inside the channel is supposed to be non-homogeneous, with the highest shear rate located near the inlet of the channel. The sheared dispersions were collected upon immersion of the outlet into water so as to avoid contact with air and then measured by SALS.

\section{Results and Discussion}

Stability Diagram with Respect to Surfactant Concentration. The stability of the colloidal system has been studied by means of SALS according to the procedure described before. The measured average structure factor, plotted according to eq 6 , gives, from the slope of the linear plot, the term $x /(1-x)$. This latter one can be plotted as a function of time, thus resulting in a linear curve, as predicted by the second-order kinetic model for the aggregation of primary particles giving doublets. As an example, linear plots of this kind are shown in Figure 4 for different values of surfactant concentration.

From the linear fit to the curves, the aggregation kinetic constant for doublet formation is evaluated, according to eq 3 . Given the good quality of the linear fit, the value of the Fuchs stability ratio can be evaluated with good precision by means of $W=K_{\mathrm{B}} / K_{1,1}$. It is worth noting that eq 6 was derived by assuming that the structure factor of the doublets can be described by the Rayleigh-Debye-Gans (RDG) theory of light scattering. ${ }^{24}$ This latter one is applicable within the following limits: $|m-1| \leq 1$ and $\left(4 \pi R_{\mathrm{p}} / \lambda\right)|m-1| \leq 1$, where $m$ is the ratio of the refractive index of the medium relative to that of the liquid ( $m=1.180$, in our case). Given the size of our primary particles $\left(R_{\mathrm{p}}=289 \mathrm{~nm}\right)$, the RDG theory is no longer valid since the second constraint is not satisfied. Therefore, the radius of primary particles is evaluated from SLS by using the LorenzMie theory, as already mentioned. Concerning eq 6 , however, the use of the RDG approximation for the structure factor of the doublet (eq 5) may be questioned. To demonstrate that the RDG expression can nevertheless be used to this scope, we show a comparison between the structure factor of a doublet calculated with the RDG theory and one calculated with the T-matrix theory (the latter one being the rigorous solution to the problem $)^{28}$ for a primary particles radius of about $289 \mathrm{~nm}$. The comparison is given in Figure 5. It can be seen that the difference between the two theories is small in the whole range from 0 to $50^{\circ}$. Since in the SALS experiments the scattering angle varies within the range 0.02 to $40^{\circ}$, by far more manageable RDG expression has been used to evaluate the kinetic constant instead of the fairly more complicated T-matrix theory. We should also mention that another accurate methodol-

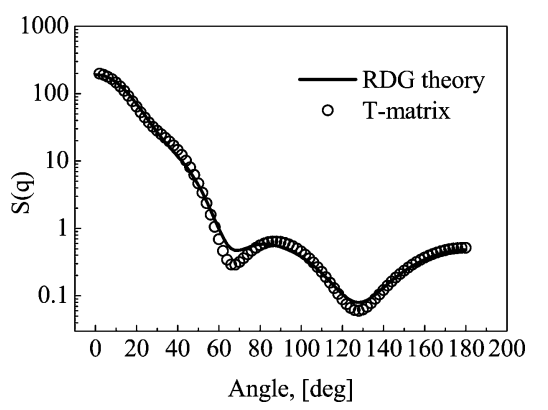

Figure 5. Comparison between the structure factor of a doublet $\left(R_{\mathrm{p}}\right.$ $=289 \mathrm{~nm}$ ) calculated with the RDG theory (line) and with the T-matrix theory (circles).

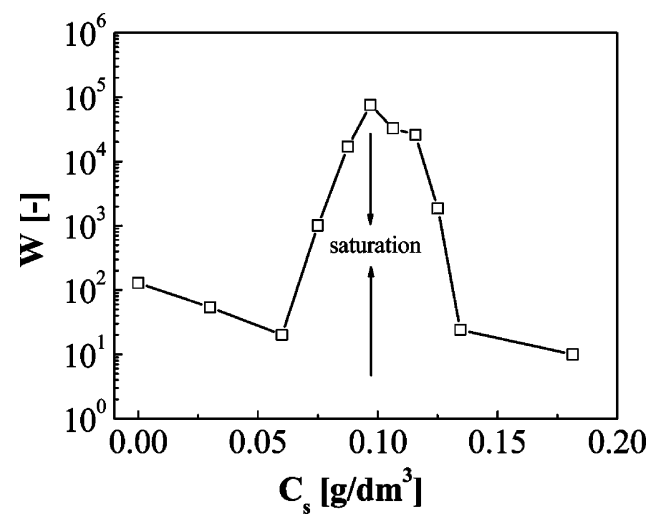

Figure 6. Stability diagram (Fuchs ratio, $W$, vs surfactant concentration, $C_{s}$ ) for the investigated system. $\phi=5 \times 10^{-3}$.

ogy to evaluate the aggregation rate constant in a total modelfree way was developed by Borkovec and co-workers, ${ }^{29,30}$ which requires a combination of simultaneous SLS and DLS measurements performed on a fiber-optics multiangle instrument.

The final output of the stability experiments at different surfactant concentration is the stability diagram of the colloidal system, represented by the Fuchs stability ratio $W$ as a function of the surfactant concentration, as shown in Figure 6. Different stability regimes, corresponding to distinct behaviors played by the surfactant, can be identified. It is useful to stress that, for the aggregation in the presence of potential energy barrier, the surfactant on the surface and in the bulk behavior plays a major role in determining the stability of the dispersion. This is reflected in the trend shown in Figure 6 where $W$ varies by several orders of magnitude with respect to variations in the total surfactant concentration in the system. In particular, it can be seen that the stability initially decreases upon addition of KS (low concentration regime), then increases steeply in the range $0.06-0.09 \mathrm{~g} / \mathrm{dm}^{3}$, passes through a maximum $\left(W \approx 10^{5}\right)$ around $0.09-010 \mathrm{~g} / \mathrm{dm}^{3}$, and finally decays rapidly up to values which are not far from the fast coagulation regime (the latter occurring at $W \approx 1$ ). A qualitative explanation of the observed trend is given in the following. Starting from bare particles of polymer, the addition of small amounts of KS leads to adsorption of the surfactant molecules onto the particles surface. As reported by Kawasaki, Ban, and Maeda, ${ }^{31}$ who studied cationic surfactant adsorbed on graphite by atomic force microscopy and surface force measurements, in the first step of adsorption, the adsorbed molecules lie down horizontally on the surface, and the charge of the head groups is neutralized by the counterion binding. Given the high affinity of the ionized carboxylic group with the $\mathrm{Mg}^{2+}$ ions used to induce aggregation, counterionspecific effects (due to van der Waals-like ion correlation attractive forces), in the present case, are expected. These are well documented in the literature for the case of negatively 
TABLE 1: Electrophoretic Mobility and $\zeta$-Potential Data Measured at Different Total Surfactant Concentration

\begin{tabular}{ccc}
\hline$C_{s}\left(\mathrm{~g} / \mathrm{dm}^{3}\right)$ & mobility $\left(10^{-8} \mathrm{~m}^{2} / \mathrm{Vs}\right)$ & $\zeta$-potential $(\mathrm{mV})$ \\
\hline 0.00 & $-2.08 \pm 0.12$ & $-26.4 \pm 1.5$ \\
0.03 & $-1.40 \pm 0.11$ & $-23.6 \pm 0.6$ \\
0.05 & $-1.90 \pm 0.08$ & $-24.9 \pm 1.0$ \\
0.09 & $-2.17 \pm 0.04$ & $-27.6 \pm 0.6$
\end{tabular}

charged lipid bilayers (KS is indeed among the model constituents of such kind of bilayers and of biological membranes), ${ }^{32,33}$ and they are most likely responsible for the observed initial decrease of $W$ with KS concentration. To support this hypothesis, $\zeta$-potential measurements have been performed in the presence of the same salt concentration for different KS concentrations. Results are reported in Table 1 and indicate that the $\zeta$-potential as well as the electrophoretic mobility also show a non-monotonic behavior: in particular, they initially decrease in absolute value upon addition of surfactant to the system. This observation may point toward a decrease of the surface charge brought about by specific counterion binding which is dominating the initial phase of adsorption and becomes less important as the surface density of surfactant molecules increases. The ion-binding effect is more visible in the initial stage of adsorption probably due to the isolated configuration of the adsorbed KS molecules (see the following paragraph for the discussion on the adsorbate morphology) that makes each of them available for the binding. Eventually this effect vanishes at higher KS concentrations (about $0.06 \mathrm{~g} / \mathrm{dm}^{3}$ ), and the main effect brought about by surfactant adsorption becomes the increase of the surface charge density. This second regime sees a dramatic increase in the stability of several orders of magnitude due to surfactant adsorption.

Increase in surfactant adsorption leads to extending surface patches covered by the surfactant on which coagulation with other particles is strongly unfavorable due to a thicker (with respect to the free patches) electric double layer (EDL) as well as to more pronounced short-range entropic effects due to the steric repulsion between surfactant molecules on approaching surfaces. ${ }^{34,35}$ The broadening of adsorbate patches with increasing KS concentration eventually arrests when saturation of the surface occurs. Once the surface available for adsorption is saturated, the molecules, due to the very low cmc of the surfactant $\left(\mathrm{cmc} \approx 10^{-5} \mathrm{M}\right)$, start associating in the form of micelles in the bulk. Micelles, in turn, give rise to well-known destabilization effects due to the attractive depletion interaction, ${ }^{36}$ thus resulting in a decrease of $\mathrm{W}$. The occurrence of saturation, in the stability diagram, corresponds to the point of maximum and allows us to make an evaluation of the density of KS molecules at the surface. In fact, we can assume that, at the concentration corresponding to maximum stability, the particles are fully covered with surfactant and that the KS concentration in the bulk phase is small compared with that on the surface of the particles. Then, under these assumptions, the KS concentration at the particles surface and the effective average surface occupied by a molecule in the adsorbate layer can be estimated. This gives about $28 \AA^{2}$ per molecule, a value which is slightly higher than the value $23 \AA^{2}$ for the area occupied by a molecule in the close-packing configuration of crystal bilayers as measured by Vincent and Skoulios ${ }^{14,15}$ by $\mathrm{X}$-ray diffraction. This observation leads to the formulation of a first hypothesis about the adsorbate layer structure which, in terms of packing density, cannot be too far from that of a monolayer, thus excluding association phenomena of mesos- copic scale. A schematic view of the main phenomena taking place on increasing the surfactant concentration is given in Figure 7.

Structure of the Surfactant Layer Adsorbed on the Particle Surface. Aiming at finding a quantitative check of the phenomenological picture inferred from the stability study, the adsorption isotherm of KS on the particles has been measured by means of surface tension measurements, according to the procedure described in the experimental section. Results are reported in Figure 8. Despite the evident difficulties encountered in carrying out measurements at very low concentrations, it is possible to appreciate a clear trend as well as to identify different adsorption regimes. The experimental data are compared in Figure 8 with a theoretical curve given by eq 11 . The fitting gave the following parameters: $n \approx 6, k_{1}=450$, and $k_{2}=2.1$ $\times 10^{8}$.

Furthermore, from the onset of the plateau (occurring at about $0.03 \mathrm{M}$ of KS) and using the following formula: ${ }^{37} A_{\mathrm{m}}=6 \mathrm{~m} /(\mathrm{c}$ $<D_{\mathrm{p}}>N_{\mathrm{A}} \rho$ ), where $m$ is the mass of polymer and $c$ are the moles of surfactant adsorbed on the particles at saturation (with $N_{\mathrm{A}}$ being Avogadro's number), it was possible to estimate the effective surface-per-molecule to be about $26 \AA^{2}$. This value is very consistent with the value $28 \AA^{2}$ evaluated from the point of maximum in the stability diagram. Finally, the transition to the plateau gives the KS concentration at which saturation of the surface is reached. In terms of total concentration of KS in the system, this is equal to about $0.095 \mathrm{~g} / \mathrm{dm}^{3}(0.03 \mathrm{mM})$, which is very consistent with the value $0.097 \mathrm{~g} / \mathrm{dm}^{3}$ found from the maximum in the stability diagram. Regarding the information about the structure of the adsorbate layer, the isotherm points toward a two-step adsorption process with a predominance of the second (cooperative) step on the first (non-cooperative) one, which is reflected in the fitting values of the respective characteristic constants of the two steps (namely, $k_{2} \gg k_{1}$ ). The value of $n$ is similar to other ones reported in the literature ${ }^{11}$ and suggests that the second step involves adsorption of bunches of, on average, 6 molecules. However, the presence of small two-dimensional surface aggregates does not mean that the adsorbate layer is a multilayer. In fact, it is more likely that, in the presence of such small hemimicelles, the layer retains a structure having geometrical features (especially in terms of packing and molecular surface density) more akin to a monolayer.

To get more insights into the structure of the adsorbate layer on the particles' surface, SLS experiments have been conducted where the average form factor of particles in the presence of different amounts of KS has been measured.

In Figure 9, as an example, the form factor of the bare particles has been plotted together with the form factor of the same particles in the presence of a total concentration of KS equal to $0.118 \mathrm{~g} / \mathrm{dm}^{3}$. The two scattering spectra are quite similar. However, we can recognize two significant differences. First of all, the second minimum for the case in the presence of $\mathrm{KS}$ is slightly shifted toward lower values of $q$. This clearly indicates a larger size of the particles in this second case with respect to the bare particles. Furthermore, the minima of the form factor measured in the presence of $\mathrm{KS}$ are deeper than in the case without KS. According to previous studies, ${ }^{38,39}$ the smoothing out of the minima from one spectrum to another might be attributed, in some cases, to increased size polydispersity as well as, equivalently, to an increased extent of surface irregularities. Since in the present case the polydispersity is the same for the two curves (the same batch of pretreated particles was used), this might suggest some change of a small extent in 


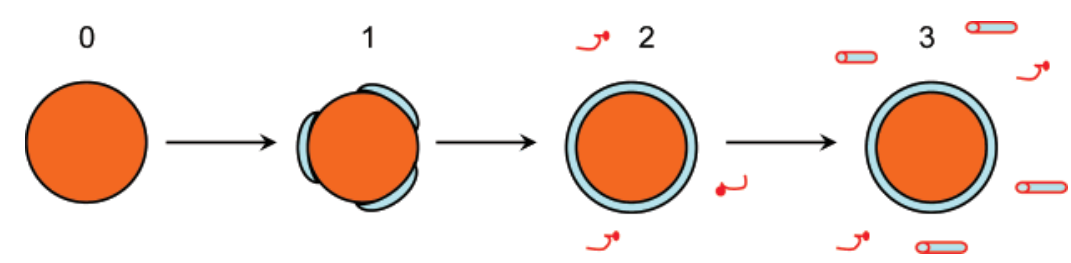

Figure 7. Schematic of the consecutive regimes encountered on increasing $C_{s}$. (0) Bare particles. (1) Partial coverage of the surface. (2) Saturation: complete coverage of the surface, free molecules in the liquid phase. (3) At increasing $C_{s} \mathrm{KS}$ molecules in the liquid form micelles.

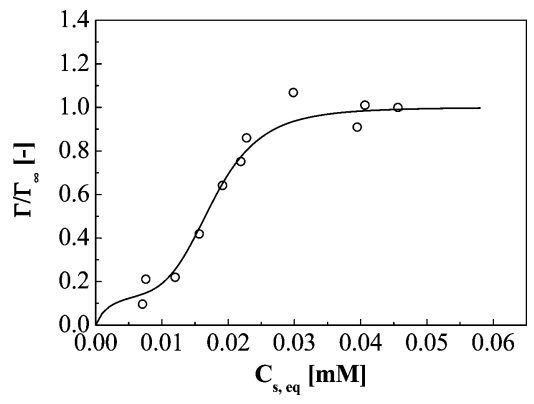

Figure 8. Relative adsorption isotherm for $\mathrm{KS}$ on the latex particles as a function of the equilibrium KS concentration in the bulk as measured from surface tension measurements. Circles: experimental data. Continuous line: theoretical fitting obtained by means of eq 11. Values of the fitting parameters are given in the text.

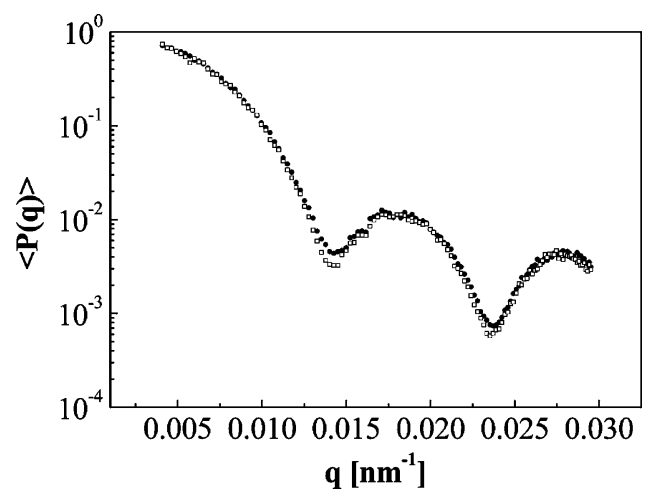

Figure 9. Scattering curves as measured by SLS for two different conditions. (๑) Bare particles without surfactant. ( $\square$ ) Particles in the presence of $C_{s}=0.118 \mathrm{~g} / \mathrm{dm}^{3}$.

surface properties. A hypothesis, thus, could be that the roughness is somehow slightly reduced in the presence of the adsorbed surfactant layer.

A quantitative fitting of the experimental scattering curves has been done by means of the Lorenz-Mie theory (eq 7), and the size polydispersity has been accounted for through the experimentally determined pdf (see eq 8 and 9), as explained in the Experiments and Methodology section. As an example, results for the case of particles in the presence of $0.118 \mathrm{~g} / \mathrm{dm}^{3}$ of KS are presented in Figure 10.

The only fitting parameter used is the diameter of the particles, the standard deviation of the PSD being fixed and equal to the value determined from SEM analysis. The same procedure has been applied for experiments at different $\mathrm{KS}$ concentration. Minimization with two fitting parameters (i.e., $\sigma$ and $R_{\mathrm{p}}$ ) including the standard deviation gives results that do not differ substantially from those obtained with the diameter as the only fitting parameter. This means, as anticipated above, that, if there are effects of reduction of the surface asperities by the surfactant, their extent is very small. The quality of the fitting was excellent in all cases. The values of the particles average diameter calculated in this way for various concentrations of $\mathrm{KS}$ are plotted in Figure 11 against the total KS concentration in the system. Despite some scattering in the data,

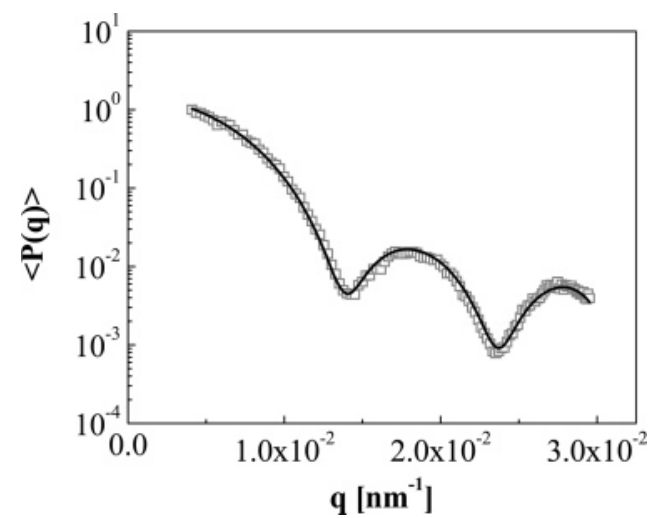

Figure 10. Normalized experimental scattering curve (squares) and fitting curve obtained with the Lorenz-Mie theory (continuous line) for the case $C_{s}=0.118 \mathrm{~g} / \mathrm{dm}^{3}$. Average particle diameter from the fitting: $\left\langle D_{\mathrm{p}}\right\rangle=581.35 \mathrm{~nm}$.

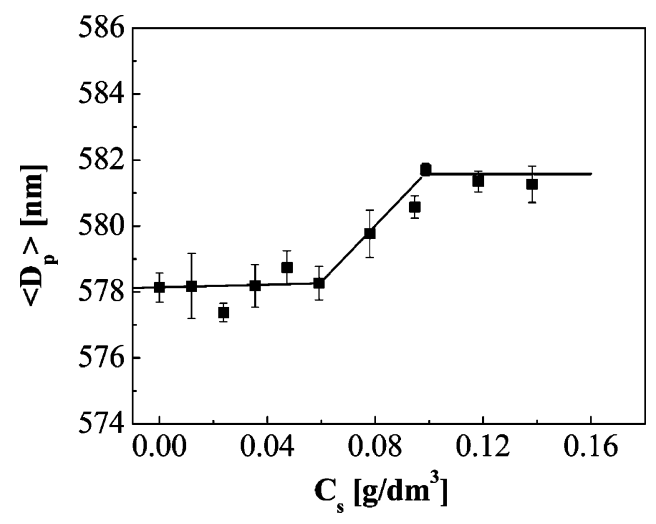

Figure 11. Average particles diameter as determined by fitting the scattering curves by means of the Lorenz-Mie theory, as a function of the total surfactant concentration in the system. The piecewise continuous line is a guide to the eye.

which is expected given the small scale of the phenomena we look at, a trend can be clearly identified. The average diameter of the particles increases on increasing the KS concentration in the system as a consequence of adsorption. The average diameter remains practically constant $\left(D_{\mathrm{p}} \approx 578 \mathrm{~nm}\right)$ in the range of low concentrations and suddenly rises at about $0.06 \mathrm{~g} / \mathrm{dm}^{3}$. Then, a second regime sets in where the diameter grows with respect to $C_{s}$, eventuallyreaching a plateau (third regime) with constant values of nearly $582 \mathrm{~nm}$. The onset of the plateau, at about $0.10 \mathrm{~g} / \mathrm{dm}^{3}$ is in good agreement with both the adsorption isotherm and the stability diagram. Notice that the concentration value at which the sudden rise is observed is not far from the value (about $0.05 \mathrm{~g} / \mathrm{dm}^{3}$ in terms of total $\mathrm{KS}$ concentration) at which the second adsorption step in the isotherm sets in. Recall that, based on the physical model of two-step hydrophobic adsorption, the first step corresponds to adsorbed single molecules horizontally aligned with the surface that thus do not contribute significantly to the growth in the particle diameter.

On the other hand, the onset of the second adsorption step is accompanied by the rising of the thickness of the adsorbate layer 


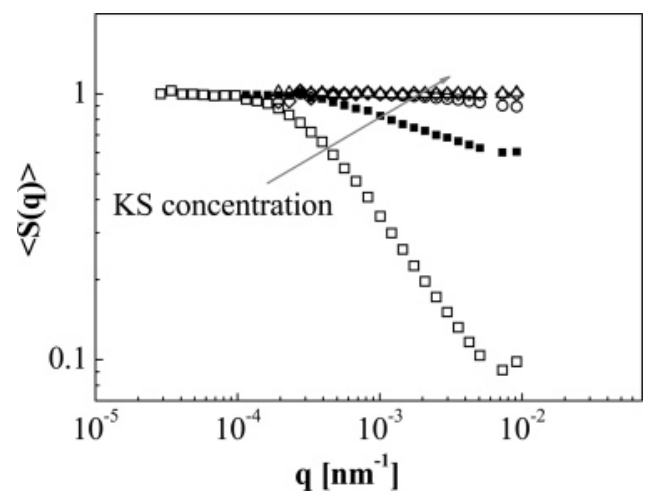

Figure 12. Normalized average structure factor of samples sheared at $\dot{\gamma}=8.15 \times 10^{5} \mathrm{~s}^{-1}$ at different values of $C_{s .}$ (口) $C_{s}=0.00 \mathrm{~g} / \mathrm{dm}^{3} ;(\mathbf{\square})$ $C_{s}=0.06 \mathrm{~g} / \mathrm{dm}^{3} ;(\bigcirc) C_{s}=0.088 \mathrm{~g} / \mathrm{dm}^{3} ;(\Delta) C_{s}=0.097 \mathrm{~g} / \mathrm{dm}^{3} ;(\diamond) C_{s}$ $=0.12 \mathrm{~g} / \mathrm{dm}^{3} ; \phi=5 \times 10^{-3}$.

and, hence, by an observable increase in the diameter of the particles. This is indeed the case, and our data seem to agree with this picture. Finally, the thickness of the adsorbate layer at saturation is almost $2 \mathrm{~nm}$. Recalling the extended length of the KS molecule $(2.5 \mathrm{~nm})$, this is in agreement with our hypotheses about morphology, since, based on this interpretation, part of the alkyl tail is in contact with the bottom molecules aligned with the surface and, being horizontally aligned itself, does not contribute to the height of the hemimicelle, which is, thus, given by a fraction of the extended length of the molecule. This can explain why the observed thickness of the layer is less than the extended length of the molecule.

Response of the System's Stability to High Fluid Shear at Different Surfactant Concentrations. Let us now discuss the results obtained by shearing dispersions containing different amounts of KS and measuring the average radius of gyration, $\left\langle R_{\mathrm{g}}\right\rangle$, of the particles/aggregates ex situ by means of SALS. The determination of $\left\langle R_{\mathrm{g}}\right\rangle$ for weakly or noninteracting scattering objects from scattering spectra is a standard procedure, and we refer the reader to previous studies. ${ }^{40,41}$ The average structure factors of dispersions at different KS content sheared at the same shear rate $\dot{\gamma}=8.15 \times 10^{5} \mathrm{~s}^{-1}$, are shown in Figure 12, which are obtained by dividing the intensity spectrum by the form factor of the primary particles as experimentally measured by the same SALS device.

It is seen that, for the bare particles without addition of KS (open squares), the structure factor indicates the presence of aggregates of considerable size. On increasing KS concentration, the slope of the curves of $\langle S(q)\rangle$ decreases, and eventually, at a surfactant concentration corresponding exactly to that of maximum stability in the (static) stability diagram, the structure factor becomes flat, thus indicating the absence of aggregates (i.e., full stability under shear).

Under shear and in the absence of salts, the driving force for aggregation is given by fluid velocity gradients resulting in hydrodynamic forces that compete with the EDL repulsion and eventually lead the particles to stick together. It turns out that, in the absence of screening electrolytes, the key parameter which, for a given system of particles, decides whether aggregation can take place or not is the amount of surfactant at the surface. In particular, when the surface is fully covered and, at least, under the conditions considered in this work, the repulsive potential uniformly distributed around the particles is such that the hydrodynamic forces cannot overwhelm it, and the dispersion is stable under shear. Instead, if there are free patches of surface not covered by surfactant, then, locally, the repulsive potential is associated with a repulsive force which is

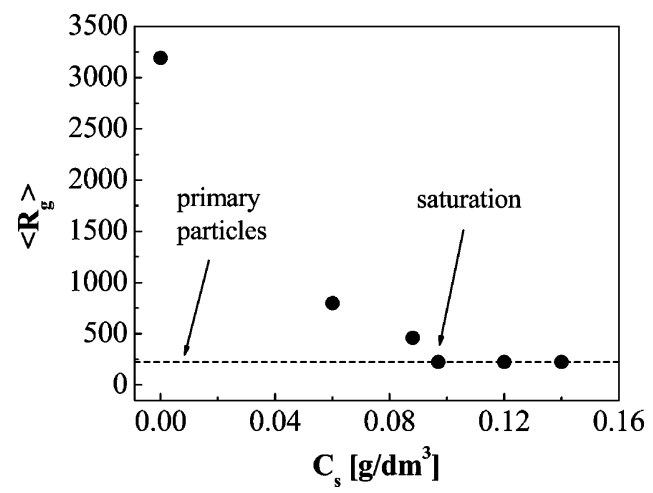

Figure 13. Average radius of gyration of the sheared samples as measured ex situ from SALS, plotted as a function of total surfactant concentration $C_{s} ; \phi=5 \times 10^{-3}$.

unable to equilibrate the hydrodynamic force pushing the particles into contact, thus resulting in aggregation.

Values of $\left\langle R_{\mathrm{g}}\right\rangle$ obtained from the Guinier plot are shown in Figure 13 as a function of KS concentration. The data demonstrate that high-shear rate aggregation in the presence of potential energy barrier is strongly affected by the amount of surfactant in the system. In particular, in the absence of surfactant, aggregation is not only possible but also leads to large aggregates made of tens to hundreds of primary particles. With increasing surfactant concentration, the potential energy barrier is increased significantly due to the adsorption of charged species, leading to a decreased collision efficiency that makes aggregation events less probable, thus resulting in a substantially smaller size of the aggregates. However, so long as there remain patches of surface free of surfactant, aggregation remains possible, although, as we have seen, its extent is reduced. This is ascribed to the fact that, even if as small as $10 \%$ of the particle's surface, a free patch at the surface is associated with a sensibly lower local interaction barrier that makes aggregation on the free patch possible. Upon adding surfactant, once saturation (full coverage) of the surface sets in, no free patches are available any longer so that the probability of overwhelming the interaction barrier is everywhere zero, thus resulting in zero collision efficiency and no aggregation phenomena are observed (the radius of gyration coincides with that of the primary particles). It should be noticed from the figure that the radius of gyration becomes equal to that of the primary particles at practically the same critical concentration of KS (about 0.097 $\mathrm{g} / \mathrm{L}$ ) that has been measured also from the stability experiments and from the adsorption isotherm as well.

A final but not less interesting consideration has to be spent about shear experiments conducted at KS concentrations higher than the critical concentration of surface saturation that means, also, in the presence of KS micelles. It is evident from the above plot that in no case does aggregation take place. This suggests that, no matter if micelles are present or not in solution, if the surfactant coverage is uniform, aggregation is not possible. In the stagnant case discussed before, in the presence of screening electrolyte, the micelles greatly affect the stability through the depletion attractive force. The latter one superimposes to the screened EDL repulsive force, eventually overwhelming it and determining a strong decrease of stability. In the high-shear case, on the other hand, since no electrolytes are present, the EDL repulsion is not screened, thus retaining a typical long-range character and a high interaction potential barrier. We can argue either that the attractive force that results from depletion and hydrodynamic gradients is not enough to overwhelm the unscreened EDL repulsion or that depletion and hydrodynamic 
forces are noncooperative. Another possibility is that depletion forces are not effective under shear conditions. This could be imputable to formation of shear-induced phenomena such as alignment of the micelles with the flow ${ }^{42}$ that might superpose to and eventually cancel out the entropic forces that are responsible for the depletion layer around the particles and, thus, for the attractive depletion forces.

\section{Conclusions}

In this work, we described the stability of a colloid-surfactant mixture in relation to its surface properties by using different techniques such as SALS, SLS, surface tension, and $\zeta$-potential measurements. The particles present an irregularly patterned surface with grains separated by depressions and represent a model system for studying colloidal dispersions as they occur in nature and applications. Stability and surface properties under both stagnant and high-shear conditions have been characterized by the above-mentioned complementary methods that proved to be effective in providing quantitative information about the effect of surfactant on stability over the entire range of surfactant concentration, as well as about the morphology of the adsorbed layer and its interaction with the particles surface.

As evidenced by initial kinetics experiments carried out by SALS, the surfactant affects greatly the stability of the particles under RLCA conditions and distinct stability regimes have been identified in the stability diagram. At surfactant concentration lower than the critical concentration for saturation of the particles surface, the adsorption of surfactant, which is a growing function of its concentration, is the main phenomenon influencing the stability of the dispersion. At saturation, the maximum stability is reached, and the saturation parameters of the adsorbed layer have been estimated and found in quantitative agreement with data from the adsorption isotherm. At concentrations higher than the critical saturation concentration, the formation of surfactant micelles in the liquid phase lead to depletion-induced destabilization effects.

By means of SLS, fitting of the form factor of the particles in the presence of different amounts of surfactant with the Lorenz-Mie theory of light scattering has made possible to estimate the growth of the adsorbed layer with respect to surfactant concentration and also in this case quantitative agreement has been found with the adsorption isotherm. Indications about the adsorption mechanism have also been drawn by this method and are found to be in agreement with the model of two-step adsorption on hydrophobic surfaces.

Finally, high-shear experiments in the absence of screening electrolytes coupled with ex situ SALS measurements gave strong evidence for the surfactant being the key parameter which determines the occurrence and the extent of aggregation under shear. In the adsorption-controlled regime, the radius of gyration of the aggregates decays with surfactant concentration and eventually becomes equal to that of the primary particles at the critical concentration for surface saturation. This implies that, at least under the conditions examined in this work, a complete homogeneous surfactant coating of the particles precludes aggregation by setting an interparticle interaction barrier all around the particles such that it cannot be overwhelmed even by extremely high hydrodynamic forces. On the other hand, the presence of small patches of surfactant-free surface causes a local decrease of the repulsive potential such that aggregation becomes possible. We also observed that, in the micellar regime, the micelles do not affect the stability under shear and the particles do not aggregate. In this regard, we speculate about possible shear alignment of micelles with the flow leading to suppression of the depletion effects.
In sum, by means of novel methodologies, we presented evidence of various phenomena that all demonstrate the crucial role of surface properties (and especially surface heterogeneities) in determining the stability of colloidal systems in the presence of inter-particle interaction barrier.

Acknowledgment. Financial support of BASF AG (Ludwigshafen, Germany) and the Swiss National Science Foundation (Grant. No. 200020-113805/1) are gratefully acknowledged. Werner Wacker, Dr. Wolfgang Schrepp, Claudia Armbrust, Dr. Brian Banaszak, and Dr. Maarten Staal of the Polymer Physics and Polymer Technology departments at BASF AG are gratefully acknowledged for having conducted the surface tension measurements. We also thank Ivano Costa for valuable help in the IDA. Dr. F. Krumeich, of EMEZ (Electron Microscopy ETH Zurich) is gratefully acknowledged for his assistance in the SEM.

\section{References and Notes} 273.

(1) Aslamazova, T.; Tauer, K. Adv. Colloid Interface Sci. 2003, 104,

(2) Otzen, D. E. Biophys. J. 2002, 83, 2219.

(3) Yin, Y.; Alivisatos, A. P. Nature 2005, 437, 664.

(4) Petekidis, G.; Galloway, L. A.; Egelhaaf, S. U.; Cates, M. E.; Poon, W. C. K. Langmuir 2002, 18, 4248.

(5) Jodar-Reyes, A. B.; Ortega-Vinuesa, J. L.; Martin-Rodriguez, A. J. Colloid Interface Sci. 2005, 282, 439.

(6) Jodar-Reyes, A. B.; Martin-Rodriguez, A.; Ortega-Vinuesa, J. L. J. Colloid Interface Sci. 2006, 298, 248.

(7) Walz, J. Y. Adv. Colloid Interface Sci. 1998, 74, 119.

(8) Feick, J. D.; Chukwumah, N.; Noel, A. E.; Velegol, D. Langmuir 2004, 20, 3090.

(9) Oberdisse, J. Curr. Opin. Colloid Interface Sci. 2007, 12, 3.

(10) Grillo, I.; Levitz, P.; Zemb, T. Eur. Phys. J. B 1999, 10, 29.

(11) Brown, W.; Zhao, J. X. Macromolecules 1993, 26, 2711.

(12) Wu, C. Macromolecules 1994, 27, 7099.

(13) Wu, C. Macromolecules 1994, 27, 298.

(14) Yang, C.; Kizhakkedathu, J. N.; Brooks, D. E.; Jin, F.; Wu, C. J. Phys. Chem. B 2004, 108, 18479.

(15) Gregory, N. W.; Tartar, H. V. J. Am. Chem. Soc. 1948, 70, 1992.

(16) Vincent, J. M.; Skoulios, A. Acta Crystallogr. 1966, 20, 432.

(17) Vincent, J. M.; Skoulios, A. Acta Crystallogr. 1966, 20, 441

(18) Jenkins, R. M.; Weiss, R. G. Langmuir 1990, 6, 1408.

(19) Lattuada, M.; Wu, H.; Morbidelli, M. Chem. Eng. Science 2004, $59,4401$.

(20) Jia, Z. C.; Gauer, C.; Wu, H.; Morbidelli, M.; Chittofrati, A.; Apostolo, M. J. Colloid Interface Sci. 2006, 302, 187.

(21) Ramkrishna, D. Population Balances; Academic Press: San Diego, 2000.

(22) Lin, M. Y.; Lindsay, H. M.; Weitz, D. A.; Klein, R.; Ball, R. C.; Meakin, P. J. Phys.: Condens. Matter 1990, 2, 3093.

(23) Wu, H.; Lattuada, M.; Sandkuhler, P.; Sefcik, J.; Morbidelli, M. Langmuir 2003, 19, 10710.

(24) Bohren, C. F.; Huffman, D. R. Absorption and Scattering of Light by Small Particles; New York: Wiley \& Sons, 1998.

(25) Glatter, O. In Neutrons, X-rays and Light: Scattering Methods Applied to Soft Condensed Matter; Elsevier: Amsterdam, 2002.

(26) Zhu, B. Y.; Gu, T. R. J. Chem. Soc., Faraday Trans. 1 1989, 85, 3813.

(27) Zhang, R.; Somasundaran, P. Adv. Colloid Interface Sci. 2006, 123, 213.

(28) Mischenko, M. I.; Hovenier, J. W.; Travis, L. D. Light Scattering by Nonspherical Particles: Theory, Measurements and Applications; Academic Press: San Diego, 2000.

(29) Holthoff, H.; Egelhaaf, S. U.; Borkovec, M.; Schurtenberger, P.; Sticher, H. Langmuir 1996, 12, 5541.

(30) Holthoff, H.; Borkovec, M.; Schurtenberger, P. Phys. Rev. E 1997, 56,6945 .

(31) Kawasaki, H.; Ban, K.; Maeda, H. J. Phys. Chem. B 2004, 108, 16746.

(32) Israelachvili, J. Intermolecular and Surface Forces; Academic Press: San Diego, 1992; Chapter 18.

(33) Bostrom, M.; Williams, D. R. M.; Ninham, B. W. Phys. Rev. Lett. 2001, 8716 .

(34) Tirado-Miranda, M.; Schmitt, A.; Callejas-Fernandez, J.; FernandezBarbero, A. Langmuir 1999, 15, 3437. 
(35) Vincent, B.; Edwards, J.; Emmett, S.; Jones, A. Colloids Surf. 1986, $18,261$.

(36) Mondainmonval, O.; Lealcalderon, F.; Phillip, J.; Bibette, J. Phys. Rev. Lett. 1995, 75, 3364.

(37) Piirma, I.; Chen, S. R. J. Colloid Interface Sci. 1980, 74, 90

(38) Jones, A. R. Prog. Energy Combust. Sci. 1999, 25, 1.
(39) Alchalabi, S. A. M.; Jones, A. R. J. Phys. D: Appl. Phys. 1995, $28,1304$.

(40) Jia, Z.; Wu, H.; Morbidelli, M. Langmuir 2007, 23, 5713.

(41) Castelletto, V.; Hamley, I. W. Biomacromolecules 2007, 8, 77.

(42) Penfold, J.; Staples, E.; Cummins, P. G. Adv. Colloid Interface Sci. 1991, 34, 451. 